\title{
Experiências espanholas e sua contribuição à rede de recursos psicossociais no Brasil
}

\author{
Magda Dimenstein \\ Universidade Federal do Rio Grande do Norte
}

\begin{abstract}
Resumo
Este trabalho visa apresentar algumas experiências e práticas no campo da atenção e reabilitação psicossocial voltadas ao portador de transtornos mentais desenvolvidos em três diferentes regiões da Espanha. Trata-se de um conjunto de ferramentas que vêm viabilizando mudanças consistentes na atenção em saúde mental, as quais se articulam em grandes eixos, pilares fundamentais do processo de reforma psiquiátrica tanto na Espanha quanto no Brasil: redefinição da gestão dos dispositivos institucionais; a perspectiva de continuidade de cuidados; atenção primária como porta de entrada e regulador do sistema; criação de unidades de retaguarda à crise e atenção às emergências e, principalmente, o investimento em programas de reabilitação e apoio comunitário contemplando alternativas de moradia e trabalho.
\end{abstract}

Palavras-chave: reforma psiquiátrica; saúde mental; reabilitação psicossocial.

\begin{abstract}
Spanish experiences and their contribution to the network of psychosocial resources in Brazil. This paper presents some experiences and practices in the field of psychosocial rehabilitation and attention directed to mental disorder developed in three different regions of Spain. This is a set of tools that make possible consistent changes in mental health care, which are linked into long lines, pillars of the psychiatric reform process both in Spain and in Brazil: redefining the management of institutional arrangements, the prospect continuity of care, primary care as the gateway and system regulator, the creation of units behind the crisis and attention to emergencies and, especially, investment in rehabilitation programs and community support contemplating alternative housing and work
\end{abstract}

Keywords: psychiatric reform; mental health; psychosocial rehabilitation.

$\mathrm{E}$ ste trabalho visa apresentar sucintamente algumas experiências e práticas no campo da atenção e reabilitação psicossocial mapeadas durante o período de estágio pósdoutoral $^{1}$ o qual teve como proposta metodológica a inserção durante um ano (agosto de 2009 a julho de 2010) na rede de saúde mental e o acompanhamento dos programas de reabilitação e apoio comunitário voltados ao portador de transtornos mentais desenvolvidos em três diferentes regiões da Espanha, a saber: Comunidade Autônoma de Madri, especificamente a área 3, correspondente à Alcalá de Henares e Torrejón de Ardoz; Girona na Catalunha e Jerez de La Frontera na Comunidade Andaluza, assim como algumas experiências em curso na capital.

Trata-se de regiões do país situadas em áreas geográficas muito distintas e que possuem especificidades em relação à rede de atenção psicossocial quanto à estruturação dos dispositivos assistenciais, ao financiamento e gestão dos recursos, à composição das equipes profissionais, dentre outros aspectos. Todavia, apresentam interfaces e pontos de contato que tornam essas experiências de reforma psiquiátrica exitosas, apesar dos problemas ainda existentes e dos desafios postos no contexto atual de crise econômica que vive a Espanha, o qual conforma um novo cenário sócio-político que tem trazido repercussões importantes ao campo do bem-estar social e das políticas públicas de saúde. Ou seja, mesmo com tantos avanços, inúmeros desafios ainda precisam ser superados, principalmente a presença do modelo asilar ou de uma lógica manicomial pulverizada agora em novas estruturas e práticas.

Apesar do cenário macropolítico desfavorável, os recursos que serão aqui apresentados fazem parte de um conjunto de estratégias que vêm viabilizando mudanças consistentes na atenção em saúde mental, as quais se articulam em grandes eixos, pilares fundamentais do processo de reforma psiquiátrica tanto na Espanha quanto no Brasil: redefinição da gestão dos dispositivos institucionais; a perspectiva de continuidade de cuidados; atenção primária como porta de entrada e regulador do sistema; criação de unidades de retaguarda à crise e atenção 
às emergências e, principalmente, o investimento em programas de reabilitação e apoio comunitário contemplando alternativas de moradia e trabalho.

\section{Gestão dos dispositivos e a perspectiva da continui- dade de cuidados em Saúde Mental}

Durante o $\mathrm{EPD}^{3}$, por meio da inserção nos diferentes dispositivos, acompanhamento sistemático das atividades cotidianas das equipes e participação nas reuniões propostas pela coordenação das áreas nas diferentes regiões da Espanha, foi possível conhecer a rede de serviços sanitários (assistência psiquiátrica/saúde mental) e sociais (reabilitação e reinserção social), articulados desde a atenção primária/AP, onde se desenvolvem programas transversais ${ }^{4}$ e longitudinais ${ }^{5}$. Isso proporcionou uma visão ampliada do processo de construção do cuidado em saúde mental, articulando o conhecimento dos recursos com as propostas assistenciais e de reabilitação em curso, bem como os desafios enfrentados pelo coletivo de profissionais, gestores, familiares e usuários que há mais de 25 anos vêm lutando pela efetivação do ideário da reforma psiquiátrica, aspectos que, seguramente, nos ajudam a pensar o contexto brasileiro.

As atividades dos serviços de saúde mental estão organizadas com base em planos cujos objetivos, prioridades, âmbito de atuação em termos geográficos e da população adscrita, os recursos materiais, humanos e financeiros necessários para o desenvolvimento das ações, bem como os critérios de avaliação e cumprimento de metas, estão claramente definidos. Essa definição é o que vem permitindo acompanhar a utilização dos recursos de forma adequada às necessidades de cada área e detectar as mudanças necessárias. Certamente, o fluxo observado nas diferentes regiões do país entre dispositivos, equipes profissionais, usuários e familiares é favorecido por certo modo de gestão e pela adoção de rotinas e procedimentos, pela operacionalização de uma política pública intersetorial, de seus princípios, propostas e recursos técnico-assistenciais. Um primeiro aspecto que chama atenção diz respeito à organização funcional da rede de atenção à saúde mental que conta com algumas comissões compostas por representantes dos diferentes financiadores, gestores, trabalhadores, usuários e familiares, as quais são responsáveis pelo desenvolvimento dos programas e supervisão dos recursos. Isso foi observado especialmente nos municípios de Alcalá de Henares e Torrejón de Ardoz, pertencentes à área 3 de Madri. Dentre essas comissões há aquelas responsáveis pelo âmbito assistencial, pela reabilitação, pela formação acadêmica e educação permanente, pelo controle de qualidade dos serviços, bem como pela definição de guias clínicas e procedimentos. Tais comissões se reúnem com periodicidade quinzenal ou mensal.

Nesse período foi possível participar de algumas reuniões, em diferentes comissões. Em que pese o risco da burocratização, do engessamento do cotidiano, da inversão de prioridades, dando-se mais importância e dedicando-se mais tempo a esses espaços de articulação que ao cuidado direto ao usuário e às necessidades dos familiares, as reuniões desses coletivos constituem-se claramente como uma potente ferramenta de articulação entre os diferentes serviços e equipes, fundamentais para a constituição da co-responsabilização e otimização dos recursos disponíveis na área. No diagrama das relações de poder macropolíticas onde se inserem gestores, trabalhadores, usuários, financiadores, etc., a gestão e seus artefatos - como as comissões - são dispositivos para ir avançando nos processos de negociação e diálogo, articulando os diferentes interesses em jogo, tentando definir linhas de ação que produzam inovações nos modos de funcionamento das estruturas institucionais.

Sabemos que a falta de planejamento e de participação dos trabalhadores de saúde, bem como dos usuários nos processos decisórios, é um fator corrente e problemático, e em Natal, especificamente, isso é muito evidente. A flexibilidade nos processos de trabalho para a "criação de múltiplas e diversas estratégias de cuidado aumentando a responsabilidade de cada profissional, não apenas nas decisões e nas competências para o projeto de cuidados, mas também na gestão dos dispositivos institucionais", é imprescindível (Yasui \& Costa-Rosa, 2008, p.35). Nesse sentido, a experiência espanhola é muito elucidativa e traz importantes contribuições em termos dos benefícios da organização e do planejamento das ações e metas, bem como seu acompanhamento, seja para toda a rede, seja internamente, em cada serviço ou equipe. Observou-se que o papel do gestor e do coordenador do serviço é decisivo para fomentar os processos de discussão, pactuação e mudança. Trabalhar com base em novos modos de gestão, pactuar ações, fomentar redes de valorização do trabalho e do trabalhador - já que os modos de cuidar estão intimamente relacionados com o grau de implicação desses coletivos - é, pois, algo visível no território pesquisado e o primeiro ponto a ser destacado dessa realidade.

Uma ferramenta importante por ser uma dos articuladores mais importantes de todo o processo de trabalho territorial e comunitário na Espanha é o Programa de Cuidados Continuados/ PCC e as correspondentes Equipes de Continuidade de Cuidados/ ECC e de Apoio Social e Comunitário/EASC. Eles foram criados para assegurar uma atenção integral a portadores de transtornos mentais graves partir da organização e coordenação dos diferentes recursos sanitários e sociais disponíveis em uma determinado território, garantindo a integração dos serviços de tratamento, reabilitação e suporte social. Trata-se, portanto, de uma forma operativa de organizar e coordenar o trabalho tanto na rede assistencial, evitando-se a fragmentação entre os diferentes serviços e a dispersão do usuário na rede, quanto nos espaços comunitários, junto aos dispositivos de apoio social, como alojamentos, comedores, oportunidades de trabalho, etc. $\mathrm{O}$ PCC tem ainda a função de garantir a manutenção dos cuidados tanto longitudinalmente (ao longo da vida de um usuário) quanto transversalmente (entre os serviços de uma rede), de favorecer a adesão ao tratamento, promover alternativas de atenção à crise, reduzindo a necessidade de hospitalização e, de melhorar o funcionamento social dos usuários e suas famílias. Para tanto, possui algumas características: flexibilidade na oferta de cuidados, sem exigências de adaptação a padrões fixos; trabalho em equipe; aposta na participação ativa do usuário no processo de elaboração do seu plano individual de cuidados e o apoio à integração social com foco na reabilitação psicossocial.

O PCC é posto em prática no Centro de Saúde Mental/CSM pelas Equipes de Cuidado Continuado ${ }^{6}$ que são responsáveis pela 
elaboração e acompanhamento de um plano individualizado de intervenção ou Plano de Cuidados Continuados que envolve o acolhimento, avaliação, definição das estratégias de derivação para outros dispositivos de saúde mental e seguimento dos processos em curso, inclusive, definindo critérios para a lista de espera e alta. Ou seja, é o elemento articulador dessa rede ampliada de cuidados. As equipes de Apoio Social e Comunitário $^{7}$ são parceiras fundamentais nesse processo e constituem uma estratégia de atenção domiciliar e social. Têm como meta proporcionar atividades de suporte e estruturação da vida cotidiana para usuários graves e crônicos, bem como de apoio e alívio da sobrecarga das famílias. Suas ações prioritárias são realizadas no entorno familiar e comunitário, evitando o isolamento, abandono e o risco de institucionalização. Além disso, colaboram para melhorar o vínculo do usuário à rede de atenção em saúde mental e de serviços sociais.

No período de permanência nos serviços foi possível viver o cotidiano dessas equipes e acompanhar o trabalho desde a elaboração dos planos individualizados de cuidados, das reuniões com as equipes dos diferentes recursos, até as visitas domiciliares e atividades realizadas pelos usuários. Alguns pontos importantes foram observados em termos do funcionamento dessas equipes, a saber:

- A dinâmica cotidiana da ECC consiste em receber os pacientes derivados do psiquiatra e psicólogo, elaborar um plano de continuidade de cuidados com base nas necessidades identificadas pelos profissionais acima, bem como realizar o acompanhamento dos casos já estão inseridos no PCC. Cada profissional da equipe fica responsável por um grupo de usuários. Além disso, realiza grupo terapêutico semanalmente, o qual tem muitos participantes e funciona como grupo aberto de suporte mútuo.

- Há reuniões periódicas, quase semanais entre a ECC e os diferentes dispositivos de atenção psicossocial, seja no CSM, seja nos distintos serviços. São encontros que visam avaliar a condição dos usuários, a adequação dos planos de continuidade de cuidados, as novas necessidades e demandas, o fluxo da rede e definir novas estratégias de cuidado. Nota-se uma forte articulação entre essas equipes que compartilham, de fato, as responsabilidades e discutem encaminhamentos conjuntamente, aspectos que na nossa realidade são claramente deficitários. Participam dessas reuniões os membros das equipes envolvidas, mas não há presença de usuários ou familiares, indicando a fragilidade dos mecanismos de controle social e empoderamento desses atores. Entretanto, psiquiatras e psicólogos do CSM também não participaram de nenhuma delas, situação que indica a dificuldade, presente na realidade espanhola e brasileira, de desenvolver a co-responsabilidade, a qualificação dos vínculos interprofissionais e a produção de práticas voltadas às necessidades dos usuários. Ademais, revela os conflitos existentes e "as várias tentativas de 'usurpar' competências e de ‘imperialismo’ entre as profissões”, tal como ressaltou Vasconcelos (1997, p. 29) confirmando o fato de que as hierarquias entre os profissionais "psi" e destes em relação às outras categorias é uma realidade em outras culturas.
- Devido à grande demanda, há uma lista de espera considerável de usuários para serem derivados para os outros dispositivos de atenção psicossocial, os quais estão sempre trabalhando no limite de sua capacidade. Tal situação aponta para a permanência indevida de usuários que já poderiam estar frequentando a rede normalizada de serviços e para a falta de uma equipe transitória que possa dar suporte às pessoas que estão com possibilidades de saída, mas que ainda necessitam vinculação com algum dispositivo. Esse cenário é observado no Brasil com os impasses relativos à cronificação e alta nos CAPS.

- Observou-se também que a ECC tem parte da sua função comprometida, pois está sobrecarregada e tem poucas possibilidades de ação fora do CSM, de acompanhar o usuário no seu entorno, de fazer conexões com os outros dispositivos. Por razões distintas, observa-se no Brasil as dificuldades vividas pelas equipes vinculadas aos CAPS de operar o matriciamento com as equipes de atenção primária ${ }^{8}$, a interlocução com a rede de saúde e a produção de cuidados extra-muros.

- A EASC tem a mesma dinâmica de reuniões mensais com cada dispositivo da rede. Discutem o encaminhamento dos casos, reorganizam o plano de continuidade de cuidados e são definidas novas estratégias de intervenção junto ao usuário, família e entorno. Essa equipe tem um cotidiano de trabalho intenso organizado em função das necessidades dos usuários. No acompanhamento diário observam aspectos tais como: cuidado e higiene pessoal, organização e limpeza da casa, lixo, lavagem de roupa, administração de remédios, uso do dinheiro, compra de cigarros, ensinam técnicas básicas de cozinha, auxiliam na ida ao CSM quando têm consultas ou ao serviço social, articulam atividades com escolas e centros comunitários, vão ao banco, etc. Ou seja, realizam atividades de suporte social, de forma que cada usuário conta com apoio suficiente para realizar as tarefas do cotidiano e se manter vinculado ao serviço.

- O trabalho com familiares é realizado pelas equipes vinculadas aos diferentes dispositivos da rede como Centro de Dia, Centro de Reabilitação Psicossocial/CRPS, miniresidência, mas é feito especialmente pelas EASC. Durante a avaliação do usuário são mapeadas as condições do entorno e da família, bem como são desenvolvidas estratégias de vínculo da mesma com o serviço. No retorno do usuário também são elaboradas estratégias de volta para casa, sendo que todas são cuidadosamente acompanhadas pela equipe. Esse trabalho de suporte às famílias tem sido um dos pilares mais importantes do trabalho de reabilitação psicossocial, pois se compreende que os portadores de transtorno mental "produzem nas famílias o sentimento de desamparo, descuido, incapacidade de prestar um cuidado adequado e despreparo para enfrentar situações de crise e o manejo do cotidiano" (Dimenstein, Sales, Galvão, \& Severo, 2010a, p. 1215) e que as mesmas, enquanto parte fundamental do processo de reinserção social, necessitam de informações sobre diagnóstico, medicação e crise, de estratégias práticas de cuidado no dia-a-dia e de vínculo com a equipe. 
- Outro aspecto interessante é o trabalho desenvolvido pela equipe EASC com

voluntários da comunidade, ação praticamente desconhecida na nossa rede de saúde mental. As formas de busca são: divulgação da atividade de voluntariado por meio de cartazes em centros de saúde, escolas, igrejas, centros comunitários; através de convênios com instituições filantrópicas e com a universidade, cujas horas de voluntariado valem créditos. A orientação ao voluntariado consiste na: apresentação da proposta e entrega de material explicativo sobre o serviço, seu funcionamento, atividades que necessitam incrementar; orientação básica sobre transtornos mentais, havendo um guia simplificado para familiares sobre esquizofrenia; indicação dos usuários que podem participar e definição das atividades: o que, quando e onde. O acompanhamento do trabalho voluntário é feito de forma sistemática após cada atividade.

- Por fim, é preciso destacar a relação dessas equipes com a rede de atenção primária. Na área 3, como em toda Espanha, a atenção primária é a organizadora dos fluxos, configurando-se como a porta de entrada dos usuários na rede. O "Programa de Enlace con Atención Primária" da área tem como objetivos: 1. Aumentar a sensibilidade dos profissionais na detecção dos problemas de saúde mental; 2. Apoiar tais profissionais no tratamento e seguimento desses problemas; 3. Evitar a fragmentação entre ações de saúde em geral e saúde mental; 4. Desenvolver, garantir e consolidar uma assistência integrada e continuada.

O Centro de Saúde Mental é a base de recepção e derivação para a rede de saúde mental. Os usuários são encaminhados pelo médico generalista para psiquiatras e psicólogos do CSM de referência e estes encaminham para a Equipe de Cuidados Continuados/ECC. Os Coordenadores dos Programas de Continuidade de Cuidados consultados assinalam que é visível a falta de conhecimento dos profissionais da atenção primária em relação às propostas da saúde mental e que há problemas no acolhimento e regulação dos fluxos, indicando que o médico generalista precisa ter uma formação mais adequada em relação à detecção precoce, uso e monitoramento de psico-fármacos, comorbidades com outras substâncias, necessitando da retaguarda especializada.

Nota-se também que, apesar dos esforços, há um matriciamento precário e reuniões pouco sistemáticas entre esses dois níveis de atenção; que não há derivação dos casos que necessitam atenção especializada, mas a prática de encaminhamentos sem critérios, gerando excessiva demanda/ lista de espera para as equipes de saúde mental. Em função dessa realidade, estão desenvolvendo estratégias para assessorar as equipes de atenção primária nos casos clínicos, construir e aplicar protocolos de uso comum, desenvolver atividades de prevenção em relação às "patologias menores", reduzindo o risco de medicalização do cotidiano, promover programas de formação continuada e de cuidado ao cuidador, além de discutir periodicamente os critérios de derivação de pacientes da atenção primária para os serviços de saúde mental por prioridade e patologia, aspectos que também necessitam ser trabalhados em nossa realidade e que dizem respeito ao desenvolvimento de uma nova cultura técnica, organizativa e ética.

Além disso, observamos outros pontos importantes: centralidade do psiquiatra na rede, com pouca comunicação presencial com profissionais dos outros dispositivos; o saber técnico supervalorizado em detrimento do saber do usuário; o mandato social de cada profissional bem estabelecido e pouco questionado, as interfaces entre os diferentes saberes são "indesejadas". Em outras palavras, apesar da existência no país de iniciativas de articulação da atenção primária com a especializada que visam organizar e regular as atividades para uma efetiva coordenação entre os dispositivos de atenção primária e saúde mental, observa-se fragilidade nesse aspecto e uma dificuldade de pôr as propostas em prática, por todos os motivos acima explicitados, que não são específicos da realidade espanhola, mas absolutamente identificáveis no Brasil, tal como atesta a vasta literatura do campo (Dimenstein et al., 2009; Dimenstein, Sales, Galvão, \& Severo, 2010; Dimenstein, Nunes et al, 2010; Dimenstein, Sales, Lima, \& Severo, 2011; Camuri \& Dimenstein, 2010 ; Barros \& Dimenstein, 2010).

Contudo, apesar de alguns pontos que indicam certo estrangulamento do processo de trabalho, de cuidado e articulação entre os serviços, fica evidente que as ECC e EASC são dispositivos estratégico para intervenções em saúde mental em virtude de estarem focadas no eixo territorial e comunitário e de serem capazes de produzir respostas às variadas demandas de saúde, sociais e institucionais dos usuários. Nesse sentido, constituem-se como práticas inovadoras desenvolvidas pelos serviços espanhóis para sustentar o processo de reforma psiquiátrica local e enfrentar o que alguns autores denominam de

crise de operatividade dos serviços, que no geral, tendem a não responderem satisfatoriamente as demandas e necessidades dos usuários, apresentam uma organização fragmentada em procedimentos e competências das diversas corporações profissionais que os compõem, não desenvolvem ações territoriais, nem definem claramente prioridades assistenciais e níveis de responsabilidade sobre a população sob seus cuidados (Mângia \& Muramoto, 2009, p. 119).

Dessa forma, servem como exemplos de boas práticas em saúde mental que podem ser adotadas em diferentes realidades como é o caso do Brasil, na medida em que estão orientados pelos mesmos princípios da Estratégia da Atenção Psicossocial que norteia nossa Política Nacional de Saúde Mental, e condizentes com as proposições da atenção primária, via Estratégia de Saúde da Família/ESF, do Programa de Agentes Comunitários de Saúde/PACS e do Núcleo de Apoio à Saúde da Família/NASF.

\section{Recursos residenciais: diversidade no acolhimento em saúde mental}

Diferentemente do Brasil, a Espanha dispõe de uma diversidade de recursos residenciais com especificidades em cada região do país ${ }^{10}$. Esses recursos contemplam os mais variados tipos de necessidade dos usuários, levam em conta o grau de autonomia dos mesmos e têm equipes técnicas que funcionam de maneira variável com tais características. Nas três regiões do país foi possível conhecer recursos tais como as mini-residências, os "pisos supervisados", "pisos concertados", além das "plazas 
de respiro familiar" e "estancias diurnas en dispositivos residenciales", bem como subprogramas de atenção domiciliar, estes últimos específicos da Andaluzia, os quais incluem distintos tipos de intervenções de apoio no domicílio do usuário. Nesse trabalho, destacamos a experiencia das mini-residências e dos pisos supervisados pelo potencial que apresentam de operarem a reabilitação psicosocial, segundo a perspectiva apresentada por Lussi, Pereira, \& Pereira Junior (2006) com base nas proposições de Benedetto Saraceno:

O processo de reabilitação consiste em "reconstrução, um exercício pleno de cidadania e, também, de plena contratualidade nos três grandes cenários: hábitat, rede social e trabalho com valor social" (Saraceno, 1996). A reabilitação psicossocial também pode ser considerada como um "processo pelo qual se facilita ao indivíduo com limitações, a restauração no melhor nível possível de autonomia de suas funções na comunidade" (Pitta, 1993). Ainda se pode definir "reabilitar" como "melhorar as capacidades das pessoas com transtornos mentais no que se refere à vida, aprendizagem, trabalho, socialização e adaptação de forma mais normalizada possível" (Liberman, 1993). (p. 450).

As mini-residências/MR são estruturas de transição que têm como proposta o acolhimento temporário de usuários para a aquisição de habilidades e competências para uma vida autônoma ou no ambiente familiar. Não são apenas destinadas para egressos de longas internações como é o caso das Residências Terapêuticas existentes no Brasil, mas configura-se como um recurso de preparação e apoio para a reinserção familiar e/ou para a vida em estruturas mais independentes como é o caso dos pisos.

A MR funciona com 20 moradores, homens e mulheres, $24 \mathrm{hs}$ por dia. A equipe profissional é composta por um diretor, uma psicóloga, uma terapeuta ocupacional, 14 educadores sociais distribuídos nos três turnos, fins de semana e feriados, um auxiliar administrativo de meia jornada e dois auxiliares de limpeza. Essa equipe também é responsável pelo acompanhamento dos pisos. A dinâmica da MR está planejada de modo a possibilitar a participação dos moradores nas atividades individuais e grupais oferecidas, bem como o acesso aos serviços de saúde, centros de reabilitação psicossocial e recursos sociais. Nos finais de semana e tardes há opções de lazer, contato com familiares e uso dos recursos recreativos comunitários como piscinas, ginástica, etc.

Todos os programas e atividades desenvolvidas na MR são organizados visando trabalhar aspectos referentes à autonomia, funcionamento psicossocial e suporte familiar nas seguintes áreas: auto-cuidado e atividades da vida diária (manejo de dinheiro, realização de compras e tarefas domésticas, hábitos alimentares); educação para a saúde que comporta informações sobre o transtorno mental, seguimento da medicação, contato com a rede de saúde mental; relações sociais que busca favorecer o manejo das interações e relações em grupo, assim como ampliar a participação do usuário em redes sociais normalizadas; lazer e integração comunitária, apoiando a realização de atividades culturais, esportivas, etc.

A seleção e o encaminhamento dos usuários para a MR são feitos nos serviços de saúde mental como um todo. Há uma comissão de seguimento e derivação que avalia a adequação da proposta e a partir disso elabora um plano individualizado de atenção residencial/PIAR estabelecendo os objetivos e proposta de intervenção. Esse plano é revisado uma vez por mês pela equipe. $\mathrm{Na}$ entrada é definido um psicólogo e um educador de referência, profissionais encarregados do seguimento e articulação com os outros serviços da rede que o usuário precisa frequentar para garantir a complementaridade das intervenções. Desde o início há uma tentativa de estabelecer alguns acordos e a participação ativa do usuário no processo de reabilitação. Nos casos onde há participação da família busca-se uma negociação constante no sentido da colaboração com o processo de recuperação. Na entrada, o usuário recebe o regulamento interno de convivência e organização do serviço, onde estão delimitados também seus direitos e deveres. Na perspectiva de fomentar a autonomia e as potencialidades de cada um se estabelece uma rotina de atividades como limpeza dos espaços comuns e do próprio quarto, uso da lavanderia, pequenas compras, etc. Estimular o apoio de um residente a outro na realização de tarefas diárias e circulação pelo bairro é também foco do trabalho. Há reuniões semanais entre a equipe e os moradores que discutem problemas de convivência, distribuição de tarefas, modificação de normas de funcionamento, etc. na perspectiva de estimular a participação ativa dos mesmos na tomada de decisões.

Trata-se, pois, de um recurso com objetivo de ser um espaço de transição, de aprendizagem para a vida autônoma, seja em seu próprio domicílio, seja em pisos. Não se trata de uma casa, mas de um serviço que prepara para a vida cotidiana. É um dos recursos mais interessantes e potentes da rede de saúde mental pela sua capacidade de alcance de uma clientela muito diversa e ampla, pois não está destinado somente às pessoas com histórico de internação psiquiátrica e que não possuem vínculos familiares e sociais. Falta no Brasil um dispositivo como esse, de caráter intermediário e transitório, onde se desenvolvem programas de reabilitação, focados no aumento da capacidade de autonomia pessoal e social do usuário, no apoio e assessoramento às famílias e coordenado com os recursos comunitários existentes na área. Nossa experiência tem sido voltada para crônicos residentes e não contempla pessoas que necessitam residências temporárias ou que não se ajustam às suas estruturas familiares.

Já os pisos supervisados/PS são recursos residenciais para quatro moradores. Os residentes contam com a supervisão e apoio dos profissionais da MR, que vão à moradia duas vezes por semana, mas pode reduzir-se a uma quando o recurso funciona de maneira mais autônoma. Cada residente paga a quantia de 90 euros mensais para cobrir gastos com alimentação, produtos de higiene e limpeza. Os pisos oferecem cobertura em termos de alojamento, manutenção diária do mobiliário e instalações, bem como cuidado e apoio à reabilitação e inserção social.

Um dos aspectos mais desafiantes do acompanhamento dos pisos, tal como observado nas visitas realizadas com a equipe, é a manutenção de uma coordenação com o resto da rede de atenção psicossocial, bem como o acesso aos recursos de atenção primária. Em segundo lugar, enfrentar os problemas oriundos da convivência, o cumprimento dos pactos estabelecidos entre os moradores e a equipe, garantir alternativas diversificadas de alimentação diária e de atividades, pois há uma tendência à manutenção de uma rotina por parte dos moradores, bem 
como realizar intervenções voltadas à aquisição e consolidação de habilidades pessoais e sociais. Em terceiro lugar, são essas equipes que lutam cotidianamente para a desconstrução da loucura como signo de aprisionamento, periculosidade e isolamento, pois apostam na convivência urbana dos "loucos" como cidadãos e buscam fazer articulação com a vida "lá fora" e construir efetivamente redes de conexão e diálogo nos diferentes espaços onde o usuário circula e constrói sua vida. Assim, a ênfase do cuidado não recai apenas no processo de cura no sentido da vida produtiva, mas no projeto de invenção da saúde e de reprodução social através da utilização dos espaços coletivos de convivência e sociabilidade. Na realidade brasileira também não dispomos desse recurso, ideal para pessoas que se beneficiariam de estruturas mais independentes, por apresentar certo grau de estabilidade psicopatológica e de autonomia, não ter enfermidades físicas ou problemas graves de mobilidade, ser capazes de pactuar normas de convivência e de funcionamento do recurso, e não necessitar de monitoramento $24 \mathrm{hs}$ por dia, tal como ocorre nos SRTs.

\section{Dispositivos de intervenção à crise, os serviços de urgência e hospitalização breve}

Quanto aos dispositivos de intervenção à crise, as diversas regiões da Espanha contam com unidades de hospitalização temporária (para agudos e sub-agudos) de curta duração, de interconsulta, hospital-dia, comunidade terapêutica (específica na Andaluzia), plantão e serviço de urgência/emergência nos hospitais gerais que funcionam de forma articulada. Tal cenário é efeito de um dos aspectos mais importantes do processo de reforma sanitária espanhola: a incorporação da psiquiatria no sistema sanitário geral (Ley 14/1986, de 25 de abril, General de Sanidad) indicando que "la hospitalización psiquiátrica debe evitarse en lo posible, ser abreviada y efectuarse progresivamente en unidades psiquiátricas de los hospitales generales de la red pública. Isso teve como consequência a criação de leitos psiquiátricos em todos os hospitais gerais do país, configurando as unidades de hospitalização breve ou de curta estância. São recursos destinados a atender urgências psiquiátricas e pessoas em situação de crise, com quadros clínicos agudos, em um tempo médio de 15 a 30 dias.

Os usuários chegam à unidade de hospitalização breve (que têm em média 15 leitos) e está situada em uma ala específica dos hospitais gerais ${ }^{11}$, encaminhados pelo serviço de urgência, pela interconsulta ou por indicação do CSM e/ou da equipe de atenção primária. Os familiares são informados e orientados acerca do processo: normas da unidade, técnico de referência e demandas de cuidados. A estrutura física é de quartos com leitos individuais, banheiro, sala para refeições, sala para terapia ocupacional, atividades em grupo ou psicoterápicas, sala da enfermagem, sala de encontro da equipe profissional. A rotina dos usuários é organizada por atividades de acompanhamento psiquiátrico, das funções vitais, alimentação e visita familiar (todos os dias de 17 às 19hs). Alguns usuários têm autorização para sair da unidade ou do hospital nos fins de semana, sob anuência da família e do próprio usuário. Todos os dias há reunião de passagem entre as equipes. O enfermeiro chefe apresenta os casos e são discutidos os encaminhamentos. Quando o usuário está estabilizado é feito um plano de inserção em outros programas e recursos da rede, bem como de acompanhamento à família.

A atenção à crise é orientada alguns protocolos que ajudam na tomada de decisão na prática clínica diária das equipes, a saber: "Guía para el ingreso hospitalario por motivos de salud mental; Guía para la actuación en urgencias por motivos de salud mental; Protocolo de contención de pacientes; Guía clínica para la atención a los trastornos de la conducta alimentária" "12. As unidades de hospitalização breve, responsáveis pela atenção à crise, são estruturas fundamentais para consolidar o modelo de atenção integral e garantir uma assistência de qualidade, que implica também na adoção, cada vez mais controlada, de medidas coercitivas, de contenção física e de privação de liberdade. Daí a grande preocupação dessas equipes em manejar protocolos normalizados e procedimentos documentados nesse nível de assistência hospitalar.

Esse cenário, radicalmente diferente do Brasil, indica uma perspectiva de maior integralidade do cuidado em saúde mental, bem como das redes assistenciais. No Brasil estamos lutando pela implantação dos leitos de atenção integral em saúde mental (hospitais gerais, CAPS III, emergências, leitos dos Serviços Hospitalares de Referência para Álcool e Drogas), que se associam aos leitos de hospitais de pequeno porte, onde estes existirem. Estes leitos devem ofertar o acolhimento integral ao paciente em crise articulados e em diálogo com outros dispositivos de referência para o paciente. Apesar da ampliação de uma rede de cuidados em saúde mental que indica o aprofundamento do processo de Reforma Psiquiátrica brasileira, há que se enfrentar os inúmeros impasses que este mesmo avanço tem, paradoxalmente, produzido. O problema do atendimento à crise do paciente psiquiátrico emerge como um dos principais entraves ao avanço do processo de aprofundamento da Reforma Psiquiátrica em curso no nosso país, situação muito mais avançada na Espanha. O desafio posto atualmente está não só na desconstrução do paradigma manicomial que sustenta as práticas de atenção à crise psiquiátrica, mas especialmente, na reorganização de uma rede de cuidados a partir de outra lógica, que articule a rede SUS como um todo e, nesse sentido, o CAPS III, os hospitais gerais e de emergência, bem como o SAMU e Pronto-Atendimentos, têm lugar de destaque.

\section{Saúde mental: necessidade de cuidados continuados e integrados}

Tal como explanado ao longo desse texto vários aspectos chamam atenção na realidade espanhola em termos de reforma psiquiátrica de base comunitária, sendo suas maiores conquistas: a forte estrutura organizada em torno da atenção à crise e da hospitalização breve; o suporte às equipes de atenção primária para que disponham de recursos de detecção precoce de casos de transtornos mentais, bem como para o manejo dos transtornos comuns presentes no cotidiano das unidades, possibilitando o vínculo e o seguimento do cuidado nesse nível e, por fim, a gestão dos recursos públicos de forma autônoma e criteriosa. Estes elementos fazem com que a atenção em saúde mental no país venha ganhando contornos bem definidos na perspectiva de ir além do estabelecimento de uma rede de serviços especializados e substitutivos ao hospital psiquiátrico, projetando uma idéia de 
rede ampliada que articula, intersetorialmente, diversos recursos e atores.

Entretanto, apesar de todos esses avanços, as gestões regionais de saúde mental, de maneira diferenciada nas comunidades autônomas pesquisadas, identificam algumas ameaças importantes em relação a este processo de consolidação da rede de atenção psicossocial. O primeiro seria o déficit de financiamento da saúde mental cujo orçamento é um dos mais baixos dentro dos recursos da saúde em geral. Há insuficientes recursos oriundos das políticas de bem-estar, especialmente nesse momento onde há urgência em abrir novos serviços residenciais e de reabilitação psicossocial. Outro aspecto preocupante é o fato de que tem havido um incremento considerável da demanda nos últimos anos para a rede de saúde mental, a qual não possui ainda, capacidade de atender a contento. Avalia-se que a oferta de serviços é insuficiente, o que vem gerando uma atenção rápida ao usuário com risco de medicalização dos problemas cotidianos, da não realização de planos de cuidados individualizados, e a atenção domiciliar tem sido prejudicada pela forte demanda ambulatorial. Os serviços hospitalares estão saturados, em parte pelo efeito de porta giratória observado nessa população, faltam alternativas para o problema da longa permanência hospitalar.

Patologias emergentes como os transtornos de personalidade e patologias duais têm interferido na dinâmica das equipes e na organização dos serviços, que não têm uma condução ainda clara acerca desses problemas, e onde há uma heterogeneidade de condutas e variabilidade clínica importante. Além disso, considera-se que o incremento de transtornos neuróticos tem dificultado a atenção aos transtornos mentais graves e crônicos na rede de atenção, especialmente no nível primário. Acrescenta-se ainda a diversidade de redes sanitárias e sociais que funcionam de forma paralela e não coordenada, fragilizando as ações em diversos âmbitos. Isso acontece principalmente em relação às drogadependências.

Do ponto de vista assistencial, identificam debilidades em termos de continuidade dos cuidados: que a colaboração com a atenção primária ainda não é suficiente para impactar nos níveis de resolutividade, o que pode ser visto no incremento das filas de espera; há pouca detecção de problemas graves nesse nível e um incremento da prescrição farmacológica e gasto farmacêutico; há poucas tecnologias psicológicas e sociais transferidas para estas equipes, que também contam com participação tímida da enfermagem em saúde mental. Observa-se que os Centros de Dia desenvolvem poucas ações voltadas à reabilitação psicossocial, sendo que a população adolescente é a mais crítica no sentido da falta de alternativas laborais, como também residenciais.

Em termos dos recursos humanos, falta uma planificação clara da necessidade de profissionais como psiquiatras, psicólogos e enfermeiros, especializados em saúde mental e formados na perspectiva do modelo público. Há necessidade de uma melhor distribuição dos profissionais no território; o enfrentamento da desmotivação e da síndrome de Burnout, frequente nas equipes, e solucionar problemas referentes à carreira e remuneração. Em relação à gestão, é necessário investir em sistemas de informação que viabilize ter a histórica clínica acessível e incrementar uma cultura de avaliação continuada das práticas e serviços. Outro aspecto problemático diz respeito a pouca participação dos usuários e familiares nos processos de tomada de decisão e a falta de implicação comunitária. Apesar dos claros avanços e conquistas em termos da concretização de políticas e iniciativas voltadas à inserção social e laboral, nota-se que a questão do controle social, do empoderamento de usuários e familiares, precisa avançar a fim de garantir a efetiva participação destes no processo de construção, avaliação e fiscalização das políticas públicas locais, bem como dos planos de cuidado.

Ao discutir a realidade espanhola, Desviat (2009) alerta para dois aspectos: a falta de uma cultura sanitária entre a população e as relações de poder assimétricas entre usuários e profissionais, que exercem forte determinação sobre suas necessidades de saúde e demandas de cuidados. Sem esquecer-se do lobby das indústrias farmacêuticas que vêm financiando não só várias atividades do cotidiano e a formação dos psiquiatras, mas também associações de familiares e usuários. No Brasil, isso pode ser claramente detectado na atualidade com as pressões de determinados setores sobre o governo de aprovar a Lei 9.637/98, que dispõe sobre a qualificação de entidades como organizações sociais e a lei 9.648/98 que permite a dispensa de licitação para a celebração de contratos de prestação de serviços com as organizações sociais (OS). Isso significa que postos de saúde e hospitais públicos em todo o país sejam dirigidos por entidades privadas, isto é, que prefeitos e governadores possam contratar, sem licitação, entidades privadas certificadas como OS. Com esse título, elas poderiam receber verbas públicas para administrar serviços de saúde municipais e estaduais. As OS tem sido um importante modelo privatista, empregado em alguns estados e municípios no país, o que tem levado a grandes precarizações das condições de trabalho e da prestação de serviço à população, proposta que desvirtua completamente a base do SUS. Esse cenário, entretanto, já é bastante conhecido na realidade espanhola. O processo de descentralização das competências sanitárias para as Comunidades Autônomas ocorrido há mais de 20 anos desencadeou o redimensionamento das competências de atenção dos níveis primário e especializado, bem como abriu novas formas de gestão. Segundo Sacardo, Fortes, \& Tanaka, (2010)

As novas experiências em gestão sanitária na Espanha estão marcadas pelo "paradigma" da denominada "competência gestionada", cuja idéia central é separar o financiamento e a regulação da provisão e produção de serviços. Os financiadores, mediante contratos, destinariam recursos aos provedores sanitários transferindo-lhes certo grau de riscos e, portanto, de potenciais benefícios e perdas. Os principais argumentos apresentados para desencadear o "processo de inovação" foram a importância da autonomia dos hospitais e outros provedores sanitários, o desenvolvimento da gestão clínica, a orientação dos serviços para a qualidade, a recuperação do protagonismo dos usuários e a ênfase crescente na efetividade da prática clínica, adotando-se protocolos pautados na medicina baseada em evidências. (p. 174)

Apesar de toda argumentação favorável, sabe-se que tais modelos apresentam problemas em relação, especialmente, à insatisfação salarial, à precarização dos vínculos de trabalho, piora dos níveis de qualidade dos serviços na percepção 
dos usuários (tempo de espera, qualidade da informação, instrumentos de reclamação, etc.), segundo pesquisa realizada pelo Observatório Nacional de Saúde, à falta de investimentos proporcional ao aumento de demanda de serviços e oferta de novos produtos e medicamentos, encarecimento dos custossaúde, dentre outros. Fazendo análise sobre a crise do modelo comunitário em saúde mental na Espanha na atualidade, Desviat (2010) aponta para

Las tendencias privatizadoras del sistema sanitario, en la insuficiente conciencia colectiva de la importancia de la salud pública, y en las dificultades de una clínica diferente, anclada en una psicopatología capaz de comprender la enfermedad mental en su determinación múltiple: histórica, social, orgánica y psicológica. (p. 01)

Sobre o incremento das políticas privatistas no país esclarece que isso abriu a possibilidade para distintas formas de gestão mista público/privada nas diversas Comunidades Autônomas/CA. Além da heterogeneidade em termos de gestão, financiamento, organização dos recursos de saúde mental, uso de tecnologias e mecanismos de inserção social nas CA - o que nos leva a dizer que não há uniformidade no país em termos da implementação da política de saúde mental - a gestão mista tem gerado consequências como a desarticulação da rede, a falta de mecanismos de avaliação da implantação da política e uma involução das propostas dos hospitais gerais assumirem a hospitalização psiquiátrica e converter as unidades de média e larga estância em unidades de reabilitação. Ou seja, nota-se que a tendência atual é do hospital ser o organizador da assistência.

Tal situação é mais evidente em Madri com o estabelecimento da Área Única de Saúde para os seis milhões de habitantes da capital, desmantelando completamente a proposta comunitária e fragmentando a perspectiva territorializada da saúde mental. Vários sindicatos têm se posicionado a favor da saúde pública e contra esse desaparecimento das 11 áreas sanitárias e a livre eleição do médico, insistindo que "la implantación del área única rompe principios básicos del sistema sanitario, regulados por la Ley General de Sanidad, como la prevención, la coordinación, la continuidad e integridad asistencial, "poniendo en serio riesgo el futuro de la Atención Primaria"," . Além disso, Desviat (2009) alerta: "la sanidad concertada implica además algo que no se suele tener en cuenta: que el cargo político se hace amo y señor de los profesionales de la salud" (p.1).

Nota-se, portanto, que na Espanha está havendo um claro retrocesso em termos do financiamento público da saúde, o qual tem implicações sérias em termos da sustentabilidade do Programa de Cuidados Continuados, de detecção precoce e de apoio social comunitário, da manutenção dos apoios institucionais vinculados às associações de usuários e familiares, da articulação com a atenção primária já fragilizada, da abertura de recursos residenciais e de reabilitação psicossocial, o que vem gerando internações evitáveis, uma valorização da hospitalização prolongada com a criação de novos leitos psiquiátricos em instituições privadas que se convertem nos manicômios reformados, em detrimento do fortalecimento dos programas de recuperação, movimentos que estão na direção oposta ao que atualmente se considera fundamental, segundo a OMS: mais leitos em hospitais gerais, mais serviços comunitários e de atenção domiciliar e cuidados na perspectiva da reabilitação psicossocial e de garantia da cidadania.

Em outras palavras, as dificuldades de avanços dos sistemas públicos de saúde no Brasil e Espanha e da política de saúde mental comunitária estão relacionadas à crise das políticas de bem-estar social no plano mundial, às dificuldades estruturais de financiamento da saúde pública, aos problemas históricos de ordem econômico-político-gerenciais, à ampla desmobilização/ despolitização dos movimentos sociais, à precarização dos vínculos de trabalho tanto no setor público quanto privado, à complexidade dos problemas vividos nas grandes metrópoles que implica em diferenças nos modelos de atenção e organização da rede de cuidados, ao corporativismo médico e ao avanço tecnológico da psiquiatria biomédica ${ }^{1415}$, fatores que aproximam duas realidades aparentemente distintas e se configuram como fortes obstáculos para a produção de um sistema intersetorial e integrado capaz de oferecer cuidado social personalizado e salvaguardas de direitos na comunidade. Isso leva Vasconcelos (2010) a considerar que

Em conjunturas difíceis com esta, é preciso reconhecer um primeiro aspecto importante: as conquistas feitas no processo de consolidação das políticas sociais universais em geral em nosso país, e em particular no SUS e no processo de reforma psiquiátrica, dependem fundamentalmente da presença e da ação política de movimentos, atores e forças sociais comprometidos com os interesses popular-democráticos, que pressionam de fora e ocupam os espaços possíveis de luta e gestão dentro do aparelho de Estado, para garantir o financiamento e a implantação das novas políticas e programas. (p. 3)

Além das alianças políticas, indica "a necessidade inexorável da articulação intersetorial de políticas e programas" como estratégia de enfrentamento desse cenário, que agrega um leque de problemas macro e também micro-políticos. Em função disso, Teixeira (2009) é enfático quando ressalta que

Quaisquer que sejam as transformações que se façam necessárias em determinadas "práticas", elas sempre implicam, necessariamente, transformações correlatas nos modos como os "problemas" são reconhecidos e configurados por esse “campo de práticas". Meu postulado de base, aliás, é esse: não há transformação no "campo das práticas" que não seja, indissociavelmente, transformação no "campo problemático". (p. 785)

Nessa direção, tem-se buscado propor novas questões que confrontem as formulações já estabelecidas e as tendências alinhadas ao ideário neoliberal, revigorando a dimensão reflexiva, crítica e propositiva do projeto político e social da Reforma Sanitária e Psiquiátrica. Conh (2009), preocupada com o enfraquecimento dessa dimensão nos últimos anos, alerta para o fato de estarmos sendo aplacados pela dimensão eminentemente técnica de gerência dos serviços e deixando de lado a dimensão política, social e civil de conquista da democracia, de fortalecimento coletivo e de pensar a saúde como questão social. Nesse intuito pergunta: 
Como propor, formular e pensar a questão de um projeto para a saúde, para que ela efetivamente tenha um conteúdo democrático? E conteúdo democrático não em termos meramente formais, mas da perspectiva da construção de cidadãos, para que traga consigo a democratização do Estado e o fortalecimento dos sujeitos sociais e da esfera pública. (p.46-47)

\section{Referências}

Barros, C. M., \& Dimenstein, M. (2010). O Apoio Institucional como dispositivo de reordenamento dos processos de trabalho na Atenção Básica. Estudos e Pesquisas em Psicologia (Online), 1, 48-67.

Camuri, D., \& Dimenstein, M. (2010). Processos de trabalho em saúde: práticas em saúde mental na Estratégia de Saúde da Família. Saúde e Sociedade, 19, 803-813.

Desviat, M. (2009, novembro). Conjeturas en torno a un modelo en crisis. XI Jornadas de Actualización en Psiquiatria y Salud Mental. "Crisis del modelo y modelos modelos de crisis". Manuscrito não publicado.

Desviat, M. (2010). Los avatares de una ilusión: la reforma psiquiátrica en España. Manuscrito não publicado.

Dimenstein, M., Severo, A. K. S., Brito, M., Soares, A. L., Medeiros, V. A. B., \& Bezerra, E. N. R. (2009). O Apoio Matricial em Unidades de Saúde da Família: experimentando inovações em Saúde Mental. Saúde e Sociedade, $18,63-74$.

Dimenstein, M., Sales, A. L., Galvão, E., \& Severo, A. K. (2010). Estratégia da Atenção Psicossocial e participação da família no cuidado em saúde mental. Physis, 20(4), 1209-1226.

Dimenstein, M., Neves, R., Paulon, S., Nardi, H., Bravo, O. A., Galvão, V. A. B.
M., Severo, A. K. S., \& Figueiró, R. (2010). La difícil y necesaria integración entre la Atención Básica y la Salud Mental en Brasil. Psicología Desde el Caribe, 26, 177-197.

Dimenstein, M., Sales, A. L., Lima, E. G., \& Severo, A. K. S. (2011). Estratégia da Atenção Psicossocial e participação da família no cuidado em saúde mental. Physis, 20, 1209-1226.

Lussi, I. A. O., Pereira, M. A. O., \& Pereira Junior, A. (2006). A proposta de reabilitação psicossocial de Saraceno: um modelo de auto-organização? Revista Latino-Americana de Enfermagem, 14(3), 448-456.

Mângia, E. F., \& Muramoto, M. T. (2009). Modelo de Matriz. Ferramenta para a construção de boas práticas em saúde mental comunitária. Revista de Terapia Ocupacional, 20(2), 118-125.

Ramos, F. (2010, 18 de junho). A Reforma que a Reforma Aguarda: o desafio Inadiável da Educação em Saúde Mental. Texto de Apoio à IV CNSM. Manuscrito não publicado. Disponibilizado pelo autor no grupo de discussão da Escola de Saúde Mental do Rio de Janeiro.

Sacardo, D. P., Fortes, P. A. C., \& Tanaka, O. Y. (2010). Novas Perspectivas na gestão do sistema de saúde da Espanha. Saúde e Sociedade, 19(1), 170-179.

Teixeira, R. R (2009). Humanização: transformar as práticas de saúde, radicalizando os princípios do SUS. Interface, 13(1), 785-789.

Vasconcelos, E. M. (1997). Desinstitucionalização e interdisciplinaridade em saúde mental. Cadernos do IPUB, 7, 19-41.

Vasconcelos, E. M. (2010, 10 de março). Desafios politicos no campo da saúde mental na atual conjuntura: uma contribuição ao debate da IV Conferência Nacional (Grupo de discussão online). Recuperado de www.ESAM_Rio_ Forum@yahoogrupos.com.br

Yasui, S., \& Costa-Rosa, A. (2008). A Estratégia Atenção Psicossocial: desafio na prática dos novos dispositivos de Saúde Mental. Saúde em Debate, 32(78/79/80), 27-37. 
1. Financiado com recursos da CAPES.

2. Com uso de ferramentas tais como análise documental, observação da rotina dos serviços e equipes, registrada em diário de campo, realização de entrevistas e conversas informais com trabalhadores, usuários e familiares.

3. Estágio Pós-Doutoral

4. Atenção domiciliar, coordenação e apoio com atenção primária e serviços sociais, hospitalização breve, são exemplos desses programas transversais.

5. Continuidade de cuidados; atenção ao crônico e às toxicodependências fazem parte do programa longitudinal.

6. Compostas por enfermeiros e trabalhadores sociais, com a contribuição dos psiquiatras e psicólogos vinculados ao CSM.

7. Compostas por trabalhadores sociais, psicólogos e educadores.

8. Equipes de Saúde da Família/ESF e dos Núcleos de Apoio à Saúde da Família/NASF

9. Dirección General de Hospitales - Borrador del Plan Estratégico de Salud Mental 2009-2014.

10. Tais especificidades não poderão ser tratadas nesse artigo, mas podem ser observados nos outros artigos que compõem esse dossiê.

11. No Brasil, trata-se dos leitos de atenção integral.

12. Com base no documento "Servicios de Salud Mental del Área 3" disponibilizado pela Coordenação de Saúde Mental.

13. http://www.madridiario.es/2010/Abril/madrid/madrid/186256/sindicatos-cargan-area-unica-sanitaria-libre-eleccionmedico.html

14. Essa Psiquiatria vem exercendo fortes boicotes à reforma psiquiátrica e às práticas inovadoras no mundo inteiro, tal como apontado por Benedetto Saraceno no evento realizado em sua homenagem em maio de $2010 \mathrm{em}$ Madrid. Isso coloca enormes questões em termos da formação do psiquiatra e do seu papel no processo de reforma psiquiátrica. Entretanto, trata-se não só da mudança no perfil profissional das categorias envolvidas com o trabalho da saúde, mas principalmente da transformação de tais profissionais em agentes de mudança a partir de um compromisso social perante o ideário do sistema de saúde e seus usuários.

15. Segundo Ramos (2010), o psiquiatra é o profissional que apresenta mais dificuldades em termos de sua formação para o trabalho em saúde mental no Brasil, pois "desprovido de ferramentas críticas, desconhecedor dos aspectos organizacionais e institucionais do campo da saúde pública, treinado para uma prática super especializada e enviesada em direção à pesquisa empírica, o psiquiatra recém-formado de hoje chega aos novos serviços com pouca bagagem para encarar os enormes desafios clínico-institucionais colocados à sua frente" (p.04).

Magda Dimenstein, psicóloga, doutora em Saúde Mental pelo IPUB/UFRJ, é professora do Programa de PósGraduação em Psicologia da Universidade Federal do Rio Grande do Norte. Bolsista de Produtividade CNPq. Endereço para correspondência: UFRN, CCHLA, Departamento de Psicologia. Campus Universitário s/n, Lagoa Nova. Natal/RN. Brasil. CEP: 59094-010. Telefone: (84)32153590. E-mail: mgdimenstein@gmail.com 